\title{
Generalized optimal placement of PMUs considering power system observability, communication infrastructure, and quality of service requirements
}

\author{
M. M. H. Elroby ${ }^{1}$, S. F. Mekhamer ${ }^{2}$, H. E. A. Talaat ${ }^{3}$, M. A. Moustafa. Hassan ${ }^{4}$ \\ ${ }^{1}$ Electrical Engineering Department, Faculty of Engineering, Ain Shams University, Egypt \\ ${ }^{2,3}$ Electrical Engineering Department, Future University, Egypt \\ ${ }^{4}$ Electrical Engineering Department, Cairo University, Egypt
}

\begin{tabular}{l}
\hline \hline Article Info \\
\hline Article history: \\
Received Mar 22, 2019 \\
Revised Des 2, 2019 \\
Accepted Des 10, 2019 \\
\hline Keywords: \\
Binary particle swarm \\
optimization \\
Communication infrastructure \\
Economic study for the addition \\
new data transmission paths \\
Gravitational search algorithm \\
Latency time \\
Minimum spanning tree \\
Observability \\
Phasor measurement unit \\
Reliability
\end{tabular}

\begin{abstract}
This paper presents a generalized optimal placement of Phasor Measurement Units (PMUs) considering power system observability, reliability, Communication Infrastructure (CI), and latency time associated with this CI. Moreover, the economic study for additional new data transmission paths is considered as well as the availability of predefined locations of some PMUs and the preexisting communication devices (CDs) in some buses. Two cases for the location of the Control Center Base Station (CCBS) are considered; predefined case and free selected case. The PMUs placement and their required communication network topology and channel capacity are co-optimized simultaneously. In this study, two different approaches are applied to optimize the objective function; the first approach is combined from Binary Particle Swarm Optimization-Gravitational Search Algorithm (BPSOGSA) and the Minimum Spanning Tree (MST) algorithm, while the second approach is based only on BPSOGSA. The feasibility of the proposed approaches are examined by applying it to IEEE 14-bus and IEEE 118-bus systems.
\end{abstract}

Copyright $(0) 2020$ Institute of Advanced Engineering and Science. All rights reserved.

\section{Corresponding Author:}

M. M. H. Elroby,

Electrical Engineering Department, Faculty of Engineering,

Ain Shams University, Egypt.

Email: mousaelroby@yahoo.com

\section{INTRODUCTION}

The Phasor Measurement Units has the ability to provide synchronized phasor measurements of voltage and currents, that distinguishing it from all other metering devices. It has been perceived that PMUs hold the capability of revolutionizing the way of power system monitoring and control [1]. In contrary to Remote Terminal Unit (RTU), the output sample rate of PMUs is high and may vary from 1 to 120 samples per second with synchronization accuracy less than $1 \mu \mathrm{s}$ and maximum total vector error of about $1 \%$ [2]. Because of this high sampling rate, PMUs provide large amounts of data; and consequently, they need modern communication systems with medium to high bandwidth in order to transmit their data. Mainly, the communication media of the power grid has been divided into two groups; owned and unowned ones. The first group is a part of power system elements, i.e., power line communication, Optical Power Ground wire (OPGW), and microwave communication media. While, the later didn't depend on the power system and may be of the type available to all users as an open access media or those owned by data service providing companies (such as leased line, dedicated data links, and satellites) [3]. CI should be established in the entire system for the purpose of high bandwidth data delivery and transmission. The transmission 
medium should satisfy the purpose of high bandwidth data and other requirement of Quality of Service (QoS). However, high per unit cost and challenges related to its communication system have made its judicial placement in an electric grid significant [4]. The data generated by PMUs needs a reliable and stable communication network. The OPGW is selected to be the media of the case study based on the high channel capacity, low latency time, and immunity to electromagnetic interference [5-8]. With limited annual investments, it would be desirable to add a limited number of PMUs until a final goal is achieved. Initially, there will not be enough PMUs to have a linear estimator. The attempt is to place the PMUs so that at each stage the selection satisfies some design criteria [9]. In order to obtain a sufficient amount of observability of a power system PMUs, installation sites are dispersed over a wide area. In recent years, many investigators presented different methods for finding the minimum number and optimal placement of PMUs with different degree of observability [10]. The actual subproblem is the issue of sequentially adding PMUs to a system starting with a low degree of observability and ending with complete observability with redundancy. Generally, observability analysis can be done using either a numerical approach or a topological approach [11]. In numerical observability analysis, for specific application, a network is observable if - and only if - its measurement gain matrix is of full column rank [12]. In this approach the observability usually is derived from Energy Management System (EMS) applications [13, 14]. However, for a large-scale system, the computational burden of this approach is heavy, and it sucks in the identification of the real zero diagonal elements of the matrix due to the possible errors in rounding numbers. Most papers, as a result, have gone through using the topological approaches to verify the system full observability [15]. On the other hand, the topological observability approach determines network observability strictly based on the type and location of measurements in the entire system. The topological observability analysis uses graph concepts. The network is observable topologically if - and only if - a spanning tree can be found in the graph. Readers may refer to $[10,16,17]$ for more details about topological observability analysis. Since in the power systems, measuring and its application lie within the power system studies, power system engineers mainly focus on these observability problems in their researches. On the other hand, in some researches, telecommunication engineers have paid more attention to communication systems. As a result, few researches have considered the whole domains of the observability and CI comprehensively [18]. A number of limitations while designing a communication system gives rise to need for an optimal solution that takes into account the QoS requirements such as reliability, data loads, latency time, and congestion of the communication network [19]. Many researchers considered the PMUs optimization problem as a minimization of the PMUs number. The mainly used optimization techniques are conventional such as linear integer programing [15, 20-23] or meta-heuristic such as Genetic Algorithms (GA) [24, 25], simulated annealing [4, 11, 26], tabu search [27], and binary particle swarm optimization [28]. In [14], the author assumed a pre-known installed communication infrastructure for the system and assigned a penalty factor for the case when a PMU is placed at a bus lacking CI. In other words, in this approach, communication infrastructure is also considered as a constraint. In [18], the measurement and communication infrastructures were optimally designed using the GA. They formulated and optimized this problem with a GA model in both simultaneous and independent approaches. The results indicate that while the total number of measurement devices for system observability may increase (and therefore, the observability is improved), the total cost is reduced. However, they did not introduce any specific method to evaluate the location of the CCBS. In addition, the meter placement has been carried out only for PMUs as measurement devices. Also, the authors considered that the cost of the network depends on the accumulative length of the OPGW only, and did not consider the allocation of the link capacity. Moreover, the authors did not take into account the quality of service such as latency time of the communication network, reliability, and the degree of the observability. Therefore, in the proposed approaches, the power system observability, CI requirements, system reliability, and the latency time are considered in the objective functions. We take into account the predesign requirements such as predefined locations of some PMUs and any existing CDs in some buses and CCBS location. For this study, two different approaches are used. The first approach uses BPSOGSA to search the best location of the PMUs and the channel capacity of the Communication Links (CLs) while the connection topology is done using MST algorithm. The second approach uses BPSOGSA to search the best location of the PMUs, the channel capacity of CLs, and the connection topology.

The rest of the paper is organized as follows: Section 2 describes the observability constraint. Section 3 introduce BPSOGSA. Section 4 describe Minimum Spanning Tree. Section 5 presents the quality of service. Section 6 presents the total cost calculation. Section 7 presents problem formulation and implementation, also this section discuss the systems variables and cost factors. Section 8 discuss the two approaches results. Finally, conclusions are extracted in Section 9. 


\section{OBSERVABILITY CONSTRAINT}

In general, given a PMU with unlimited number of channels at a bus, bus voltage phasor and all current phasors along lines connected to that bus will be available. As shown in (1) presents observability constraint in general form as introduced in $[10,16]$ for complete observability with a required degree of redundancy (Without Conventional Measurements - With Conventional Measurements), one depth of unobservability (Without Zero Injection Measurement - With Zero Injection Measurements)and,two depth of unobservability (Without Zero Injection Measurement - With Zero Injection Measurement ).

Observability constraint: $\mathrm{TX} \geq \mathrm{B}$

$\mathrm{T}$ and $\mathrm{B}$ are a matrix and vector depend on each case $[10,16]$.

$\mathrm{X}$ is the PMUs placement variables $\mathrm{X}=\left[\mathrm{x}_{1} \mathrm{x}_{2} \ldots \mathrm{x}_{\mathrm{n}}\right]$,

$$
x_{i}= \begin{cases}1 & \text { if PMU at bus } i, \\ 0 & \text { if No PMU at bus } i,\end{cases}
$$

The minimum number of PMUs $\left(\mathrm{PMU}_{\mathrm{Smin}}\right)$ can be formulated as a problem of Integer Linear Programming [29] as shown in the following equation.

$$
\mathrm{PMU}_{\text {Smin }}=\left\{\begin{array}{l}
\min \sum_{\mathrm{k}=1}^{\mathrm{N}} \mathrm{x}_{\mathrm{k}} \\
\text { Subject to: Observability constraint }
\end{array}\right.
$$

where

$\mathrm{N}$ is the number of buses

\section{BINARY OPTIMIZATION USING HYBRID PSO AND GSA}

The PSOGSA is a hybrid optimization algorithm, combining strengths of both PSO and GSA. This algorithm performs both PSO and GSA in terms of improved exploration and exploitation [30]. This technique has the nature of meta-heuristic optimization techniques. One of the main advantage of these techniques is that they do not need a function formulation, but rather need a fitness function only or any other way for distinguishing the results. As a result, the black box problem can be solved using these techniques. The BPSOGSA algorithm is a binary version of hybrid PSOGSA. Readers may refer to [31] for more detailsabout this algorithm. For the above-mentioned reasons, this version will be used in this study.

\section{MINIMUM SPANNING TREE}

The vertices (nodes) of the $\mathrm{CI}$ in a power grid correspond to PMUs, CDs, and CCBS, while the edges correspond to high-voltage lines [32] or a new data transmission paths. Dijkstra's algorithm, conceived by Dutch computer scientist Edsger Dijkstra in 1959 [33] is a graph search algorithm that solves the shortest path problem for a graph with nonnegative edge [34]. The Dijkstra's algorithm is used to search the short path in the MST algorithm. The complete pseudocode for MST algorithm is shown in Figure 1 Step 3 in this algorithm could be modified to start with a highest short path and end with the lowest short path. This modification is preferable when small propagation time delay is required, where this modification shrinks the network and reduces the maximum propagation time delay of the farthest site. In step 4 , the node is connected with tree through switch.

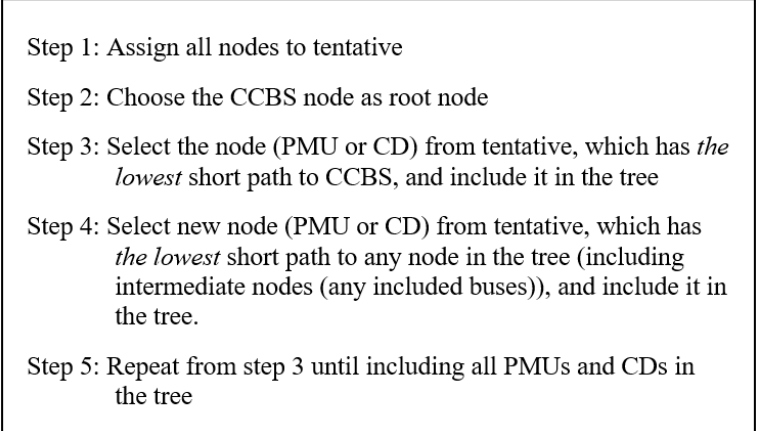

Figure 1. MST Pseudocode 


\section{QUALITY OF SERVICE}

The Wide Area Measurement System (WAMS) is a distributed communication network. The QoS in the WAMS depends on the latency time, data losses and reliability of the system. The latency time performance is very important especially in protection and dynamic control applications [35]. The tree network is a common methodology in order to design the networks [36, 37]. The network architecture consists of several PMUs, CDs, and Phasor Data Concentrators (PDCs) as shown in Figure 2. PDC collects the data generated by these PMUs over a shared communication network. Additionally, it performs quality checks on phasor data and interprets and inserts the missing data at their appropriate position [38, 39]. Typically, many PMUs located at various substations gather data and send it in real time to a PDC. Many PDCs can be connected to a common central PDC, in order to provide an interconnection wide snapshot of the power grid measurements. In large systems, they may contain more than one PDC, where each PDC is placed in a subarea. For simplicity in this study, the power system is assumed as one area, and only one PDC is used in the control center. The measurements are made at specific time instances and physical distant locations. They are then transmitted to a common location for use by wide area applications.

The latency time experienced by data between PMU and the destination node (CCBS) is a combination of PMU reporting delay, the network propagation delays, queuing, routing delays, and PDCs delay [40]. A representative latency time of the data network is shown in Figure 3. PMU reporting delay $t_{p m u}$ is defined as the maximum time interval between the data report time as indicated by the data timestamp, and the time when the data becomes available at the PMU output. This delay includes many factors, such as the window over which data is gathered to make a measurement, measurement filtering, and the PMU processing time. PDC delay $t_{p d c}$ is defined as the maximum time interval between the data input time as indicated by the data timestamp, and the time when the data becomes available at the PDC output. This delay includes many factors such as processing, and alignment received data from PMUs/PDCs. The PDC aligns received data and places that data in a packet. In addition, the PDC data processing may include filtering, reporting rate conversion, interpolation, extrapolation, phase and magnitude adjustment, etc. Most of the time the data frame is transmitted continuously from the PMU or PDC at the designated reporting rate.

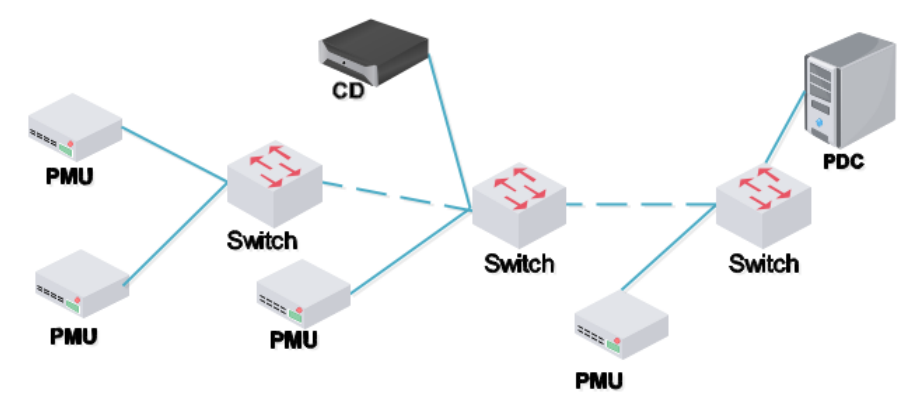

Figure 2. CDs and PMUs network

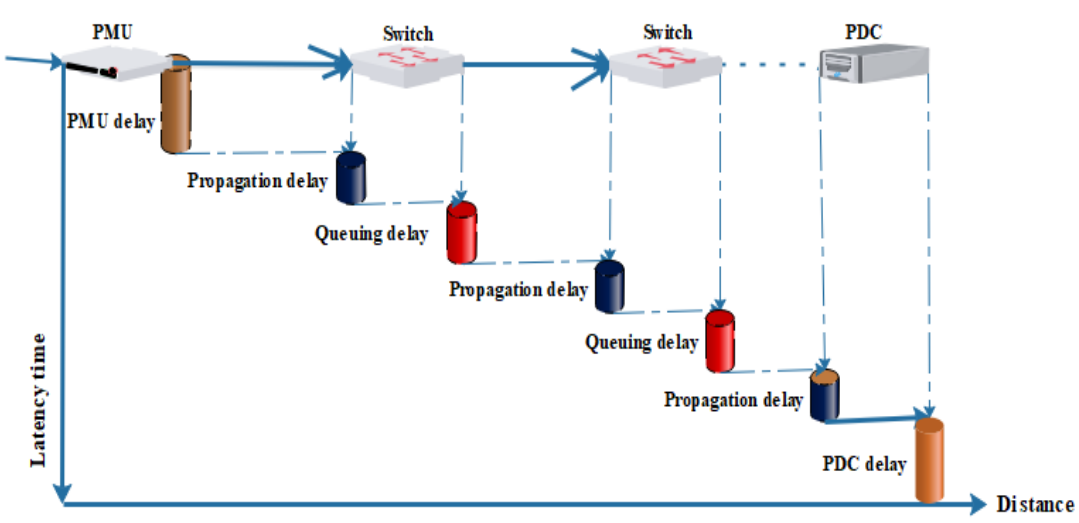

Figure 3. Latency time 
Queuing and transitions delays $\left(T_{q u}\right)$ are caused by the amount of data that has to be transported across the medium and the data rate of the medium. Considering an M/M/1 model, $T_{q u}$ can be expressed as follows [41]:

$$
\begin{aligned}
& \mathrm{T}_{\mathrm{qu}}=\frac{\mu}{C_{l}-f_{l}} \\
& \mathrm{C}_{l}>\mathrm{f}_{l}
\end{aligned}
$$

where $\mu$ is the average packet length in bits, $C_{l}$ and $f_{l}$, respectively, represent the capacity and the flow of the link 1 in bps.

The propagation delay $\left(t_{p}\right)$ is dependent on the medium and thus is a function of both the medium and the physical distance separating the individual components of WAMS. In the fiber optic, the propagation delay can be considered as in the following equation [42].

$$
t_{p}=\frac{N L}{S}
$$

where

$\mathrm{S}$ is the speed of the light in a vacuum.

$\mathrm{L}$ is the length of the communication link

$\mathrm{N}$ is the group index of the material $\approx 1.5$

Consider the network is connected using backbone switches. We can conclude the above-mentioned facts and summarize the total communication latency time as in the following equation:

$$
\mathrm{T}=\mathrm{t}_{\mathrm{pmu}}+\sum_{\mathrm{i}=1}^{l_{\mathrm{n}}} \mathrm{t}_{\mathrm{p}_{\mathrm{i}}}+\sum_{\mathrm{i}=1}^{S \mathrm{~W}_{\mathrm{n}}} \mathrm{t}_{\mathrm{qu}_{\mathrm{i}}}+\mathrm{t}_{\mathrm{pdc}} \mathrm{pdc}
$$

where

$\mathrm{T}$ is the total latency,

$l_{n}$ is the number of links between PMU and CCBS

$\mathrm{SW}_{\mathrm{n}}$ is the number of switches between PMU and CCBS

$\mathrm{t}_{\mathrm{pdc}}$ is PDC delay

Based on the typical values shown in Table C.2 in [43], and with assuming PDC uses direct forward mode $\mathrm{t}_{\mathrm{pdc}} \approx 2 \mathrm{~ms}$, and $\mathrm{t}_{\mathrm{pmu}} \approx 25 \mathrm{~ms}$. The (5) will be as follows:

$$
\mathrm{T} \approx 25+\sum_{\mathrm{i}=1}^{l_{\mathrm{n}}} \mathrm{t}_{\mathrm{p}_{\mathrm{i}}}+\sum_{\mathrm{i}=1}^{S \mathrm{~W}_{\mathrm{n}}} \mathrm{t}_{\mathrm{qu}}+2
$$

Queuing packet losses mostly occur because of the finite queue capacity of packet switching networks. To compute the average loss rate at each switch, each node is modeled with the M/M/1/k queuing system. On the topic of [44], the total number of packet losses is estimated as a function of the link flow and the capacity of buffers and links. The form of average packet loss will be:

$$
\text { Average packet loss }=\frac{1}{\sum_{\mathrm{d}} \mathrm{h}_{\mathrm{d}}} \sum_{\mathrm{e}} \frac{1-\rho_{\mathrm{e}}}{1-\rho_{\mathrm{e}}^{\mathrm{be}+1}} \rho_{\mathrm{e}}^{\mathrm{be}}
$$

where

$\rho_{\mathrm{e}}=\frac{\mathrm{f}_{\mathrm{e}}}{\mathrm{y}_{\mathrm{e}}}$

$\mathrm{h}_{\mathrm{d}}=$ Traffic volume for all PMUs and CDs

$\mathrm{b}_{\mathrm{e}}=$ Buffer capacity of link e,

$\mathrm{y}_{\mathrm{e}}=$ Capacity of link $\mathrm{e}$,

$\mathrm{f}_{\mathrm{e}}=$ Traffic flow of link e.

For a system, which contains 500 PMUs and $500 \mathrm{CDs}$ with each data flow $128 \mathrm{kbps}$ and 500 links with flow link ratio $\rho_{e}$ equal to 0.8 , if we use a buffer memory equal to one mega, average packet loss $\approx 0$. Therefore, the buffer memory can be assumed with enough value and the cost of this memory relative to other component in CI can be neglected. This leads to reducing variables in the optimization problem and reducing the run time.

The reliability of the WAMS depend on the reliability of media channel and nodes elements. Based on the concept in [45] we can describe the relation which assess the reliability of connection between any Required-node (Rnode) and CCBS as follows: 


$$
\begin{aligned}
& \mathrm{RS}=\prod_{\mathrm{S}} \mathrm{R}_{\mathrm{i}} \\
& \mathrm{Rp}=1-\prod_{\mathrm{p}}\left[1-\mathrm{R}_{\mathrm{i}}\right]
\end{aligned}
$$

where

$\mathrm{R}_{\mathrm{s}}=$ series element reliability,

$\mathrm{R}_{\mathrm{p}}=$ parallel element reliability,

$\mathrm{s}=$ Number of series elements in a path

$\mathrm{p}=$ Number of parallel elements in a paths.

For each bus reliability calculation, there are two cases: Case1) Complete observability without redundancy. In this case, there is only one path. If the PMU is located at Rnode the series components are only communication components (i.e. switches, communication links (cl), and PDCs) as shown in Figure 4(a). If the PMU is located at Neighbor-node (Nnode), the series components are communication components plus the transmission line (T.L.) as shown in Figure 4(b) (Case2) Complete observability with redundancy. In this case, there are series path and parallel paths as shown in Figure 5 and Figure 6 (for two degree of redundancy). If the PMUs are located at Rnode, the path contain Rnode has only communication components and the other paths have communication components plus T.Ls. If the PMUs are located at Nnodes, all paths contain communication components plus T.Ls.

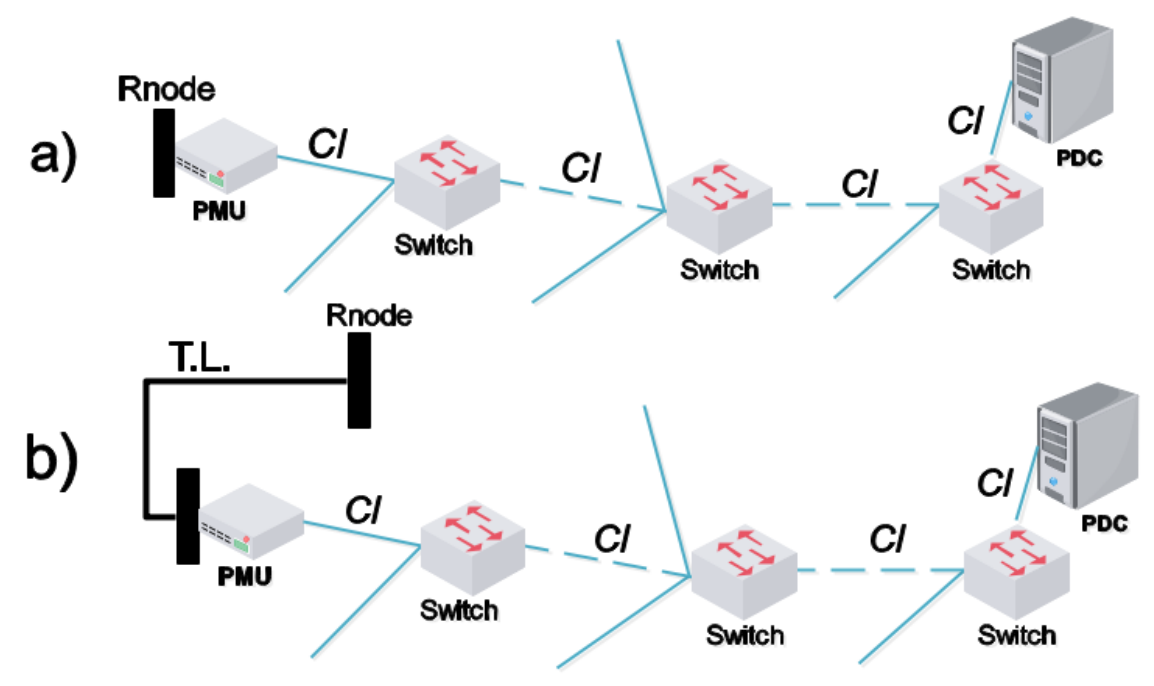

Figure 4. Complete observability

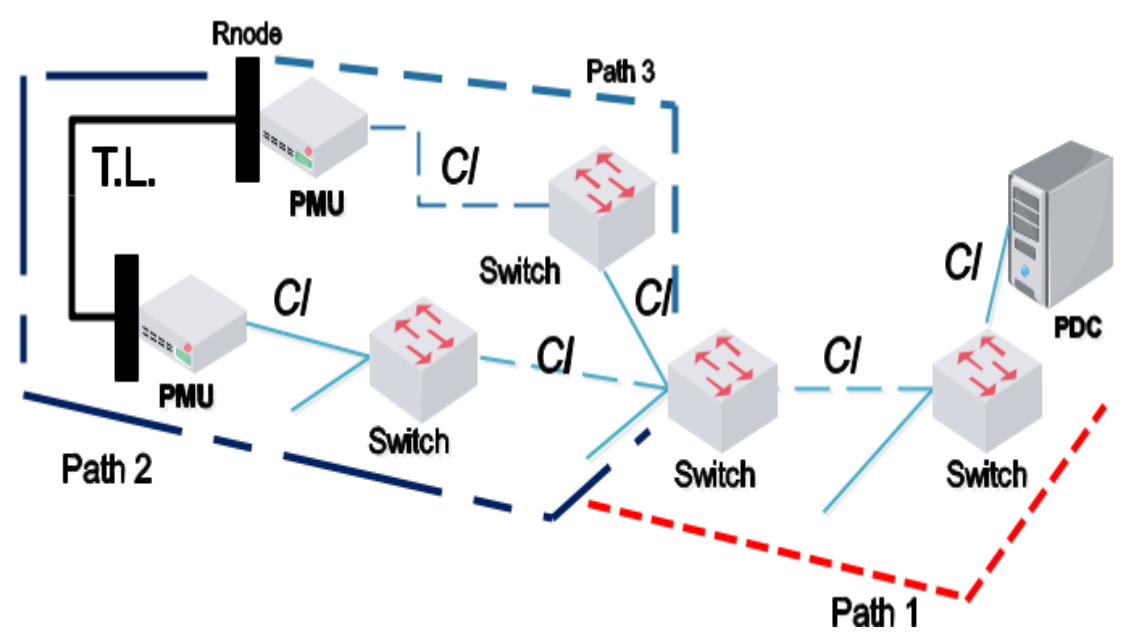

Figure 5. Redundancy with PMU at Rnode and Nnode 


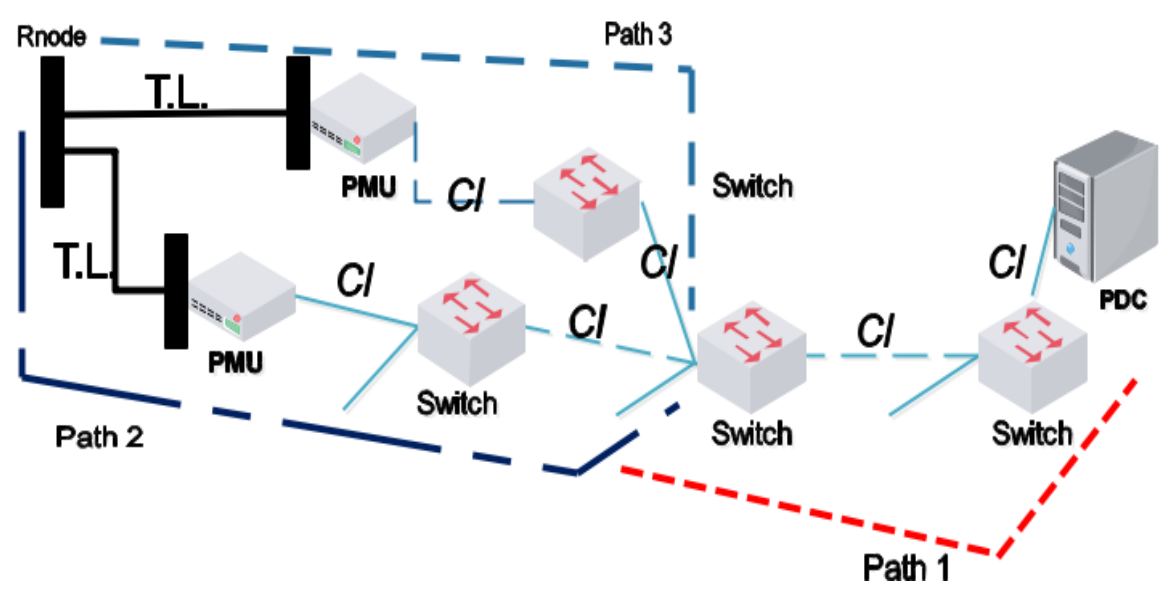

Figure 6. Redundancy with PMUs at Nnodes

In this study, the reliability of each switch is assumed as 0.99 and the reliability of OPGW and transmission lines is calculated as follows:

$$
\text { Reliability }_{\text {cl or T.L. }}=\mathrm{R}^{\mathrm{L} / \mathrm{BL}}
$$

where

$\mathrm{L}$ is the length per km of the OPGW link or transmission line

$\mathrm{BL}$ is the base length

$\mathrm{R}$ is the reliability of the base length

In this study, the base length and the reliability of the base length is assumed $20 \mathrm{~km}$, and 0.99 respectively

\section{COST CALCULATION}

The WAMS cost depends on CI cost, PMUs cost, and CCBs cost. The cost of a CI is mainly composed of two major costs including the cost of passive components and the cost of active devices. In fiber optic networks, the price of passive components mainly depends on OPGW length and capacity. On the other hand, the cost of active devices mainly depends on the number of switches, which are installed at backbone nodes [46]. As a result, the cost of CI correspond directly to the number of switches, and data transmission medium (i.e. OPGW) price and installation cost as in the following equation

$$
\operatorname{Cost}_{\mathrm{CI}}=\sum_{\mathrm{i}=1}^{l} \mathrm{~L}_{\mathrm{i}} \mathrm{d}_{\mathrm{i}}+\sum_{\mathrm{i}=1}^{\mathrm{SW}} \mathrm{SWc}_{\mathrm{i}}
$$

where

$l=$ number of the links

$\mathrm{L}_{\mathrm{i}}=\mathrm{L}_{\mathrm{crp}_{\mathrm{i}}}+\mathrm{L}_{\mathrm{in}_{\mathrm{i}}}$

$L_{c r p}=$ link capacity rate price factor (Depend on the link capacity)

$L_{i n}=$ link installation cost factor

$d_{i}=$ length of the link

$$
\text { SWc }{ }_{\mathrm{i}} \approx \text { switch }_{\operatorname{crp}_{\mathrm{i}}}+\text { switch }_{\mathrm{in}_{\mathrm{i}}}
$$

switch $_{\mathrm{crp}}=$ switch capacity rate price factor

switch $_{\text {in }}=$ switch installation cost

Subscript $\mathrm{i}$ indicate link or node $\mathrm{i}$

Actually, however, the model should be such that the price of channel capacity can take only discrete values. In addition to the CI cost, there are the cost of PMUs, which equal to the total price of the PMUs and its installation cost.

$$
\operatorname{Cost}_{\text {PMUs }}=\sum_{\mathrm{i}=1}^{\mathrm{pmu}} \text { pmuc }_{\mathrm{i}}
$$


where

pmu=number of the PMUs

pmuc $_{\mathrm{i}}=\mathrm{PMU}_{\mathrm{pchn}_{\mathrm{i}}}+\mathrm{PMU}_{\mathrm{in}_{\mathrm{i}}}$

$\mathrm{PMU}_{\mathrm{pchn}_{\mathrm{i}}}=\mathrm{PMU}$ price factor (depend on the number of channal of the PMU)

$\mathrm{PMU}_{\mathrm{in}_{\mathrm{i}}}=\mathrm{PMU}$ installation cost (depend on site location)

In the case of adding new data transmission paths between buses, the economic study has considered the establishment of new towers; the cost of the total new towers will depend on the link length. The total link cost can be calculated as follows:

$$
\begin{aligned}
& \operatorname{cd}_{\mathrm{i}}=\left(\operatorname{Lavcrp}+\mathrm{L}_{\mathrm{in}_{\mathrm{i}}}\right) \mathrm{d}_{\mathrm{i}}+\mathrm{tc}=\mathrm{L}_{\mathrm{avi}} \mathrm{d}_{\mathrm{i}}+\alpha_{\mathrm{i}} \mathrm{d}_{\mathrm{i}}=\mathrm{L}_{\mathrm{avi}} \mathrm{d}_{\mathrm{vi}} \\
& \mathrm{d}_{\mathrm{vi}}=\frac{\mathrm{d}_{\mathrm{i}}\left(\mathrm{L}_{\mathrm{avi}}+\alpha\right)}{\mathrm{L}_{\mathrm{avi}}}=\mathrm{d}_{\mathrm{i}}\left(1+\frac{\alpha_{\mathrm{i}}}{\mathrm{L}_{\mathrm{avi}}}\right)=\mathrm{d}_{\mathrm{i}}(1+\beta)
\end{aligned}
$$

where

$c d_{i}=$ cost for direct link $\mathrm{i}$

Lavcrp=Capacity rate price factor of the new data transmission paths

$L_{\text {in }}=$ link installation cost factor

$t c=$ towers cost $=\alpha d_{i}$

$\alpha=$ tower cost factor

$$
L_{\text {avi }}=\operatorname{Lavcrp}+L_{\text {in }}
$$

$d_{i}=$ actual distance for link i

$d_{v i}=$ virtual direct distance for link $\mathrm{i}$

$\beta=$ direct connection factor

The minimum number of the PMUs required could be calculated using (2). In addition, the number of CDs (Ncds) are known, so that the capacity of the new data transmission line $\mathrm{C}_{\mathrm{av}}$ ) could be approximated as follows:

$$
\mathrm{C}_{\mathrm{av}} \approx\left(\frac{\mathrm{PMU}_{\mathrm{Smin}}}{2}\right) \mathrm{PMU}_{\text {data flow }}+\left(\frac{\mathrm{Ncds}}{2}\right) \mathrm{CD}_{\text {data flow }}
$$

where

$\mathrm{PMU}_{\text {dataflow }}$ is rate of the pmu data flow (kbps)

$\mathrm{CD}_{\text {dataflow }}$ is rate of the $\mathrm{CD}$ data flow $(\mathrm{kbps})$

For each existing economic study between two buses, calculate virtual distance from (14) for all possible new data transmission paths. After calculating virtual distance there are two-distance matrices: distance matrix corresponding to transmission lines distance matrix $\left(D_{\text {power }}\right)$ and distance matrix from virtual calculating $\left(D_{\text {virtual }}\right)$. Merge the two matrixes in one matrix $D_{\text {merged }}$ as follows:

- For direct connected buses, compare the link distance in $\mathrm{D}_{\text {power }}$ with $D_{\text {virtual }}$ and take the $d_{v i}$ as link distance if it is less than $d_{i}$ in $\mathrm{D}_{\text {power }} . d_{v i}$

- For not direct connected buses, take the virtual length as link distance.

- Then modify (11) as follows:

$$
\operatorname{Cost}_{\mathrm{CI}}=\sum_{\mathrm{i}=1}^{l p} \mathrm{~L}_{\mathrm{i}} \mathrm{d}_{\mathrm{i}}+\sum_{\mathrm{i}=l p+1}^{l}\left(\mathrm{~L}_{\mathrm{i}} \mathrm{d}_{\mathrm{i}}+\alpha \mathrm{d}_{\mathrm{i}}\right)+\sum_{\mathrm{i}=1}^{\mathrm{SW}} \mathrm{SWc}_{\mathrm{i}}
$$

where

from 1 to $1 \mathrm{p}$ are the links from power system network

from $l_{p}+1$ to $l$ are the links from new added paths

Finally, the total cost will be as following

$$
\text { Total Cost }=\text { Cost }_{\mathrm{CI}}+\text { Cost }_{\mathrm{PMUs}}+\operatorname{Cost}_{\mathrm{CCBi}}
$$

where

Cost $_{\mathrm{CCBi}}$ is the cost of CCBS (Include CCBS site and PDC cost) 


\section{PROBLEM FORMULATION AND IMPLEMENTATION}

The PMUs optimal placement problem can be considered as nondeterministic polynomial complete problem [47]. For a system with $\mathrm{N}$ buses, the search space is $2^{N}$ without considering CI topology, channel capacity allocation, and number of PMU channels. Therefore, the PMUs optimal problem is considered as a combinatorial optimization problem [48]. Meta heuristic algorithm population based methods, such as BPSOGSA, are candidate for solving such problems. In the following, two approaches are presented to minimize the total cost with considering the observability and CI. In these approaches, the optimization problem is defined as follows:

$$
\text { Prob. : }\left\{\begin{aligned}
& \text { Min: }\text { (Total Cost } \left.=\text { Cost }_{\mathrm{CI}}+\text { Cost }_{\mathrm{PMUs}}++ \text { Cost }_{\mathrm{CCBi}}\right) \\
& \text { variable: PMUs locations, the network } \\
& \text { topolgy, link capcity, and buffer memory } \\
& \text { Subject to: }\left\{\begin{array}{l}
\text { i) Observability constraint } \\
\text { ii) Connection constraint (All PMUs, CDs, and } \\
\text { CCB are connected }
\end{array}\right) \\
& \text { iii) Latency time constraint } \\
& \text { V) Reliability constraint }
\end{aligned}\right.
$$

The following considerations are made in these approaches:

- Some CDs are existed, and will be connected with the communication network.

- Two Cases are considered for location of the CCBS; predefined and free selected.

- Some PMUs locations are predefined and included in the cost calculation.

- Conventional measurements and ZIB with a required degree of observability and required redundancy are considered in the observability constraint such as in Section 2

- The buffer to store the packet in the switching node has a fixed enough value and not considered in the optimization problem.

- Based on fairness grade of service, the link capacity is allocated to minimize the maximum latency time. The maximum Latency time for any $\operatorname{PMU}\left(\mathbf{t}_{\mathbf{r e q}}\right)$ is less than $0.04 \mathrm{Sec}$.

- PMU and CD dataflow are assumed $128 \mathrm{kbps}$.

- $\quad$ The reliability of the observability for any node (rreq) is greater than 0.8

In the following sections, the IEEE 14 and IEEE 118 systems with given data in below is investigated for full observability condition using PC with Intel Core i5-430M @ 2.27 GHz and Matlab 2016. In addition, the results of these approaches are compared with the method, which was presented in [18] with fixed channel capacity (ten times actual data flow) and without adding new data transmission paths. Distance matrix of each IEEE test network, we have assumed that all transmission lines have the same conductors. Thus, the relative distances between system buses can be extracted from system admittance matrix. In addition, the distances of the new data transmission paths are assumed as listed in Table 1 and Table 2 for IEEE 118 bus and for IEEE 14 bus. Table 3 and Table 4 show CCBS per unit cost at each bus for IEEE 14 bus and IEEE 118 bus systems respectively. Table 5 shows the rate of the data flow for PMU and CD. Table 6 presents cost factors values, which are used to calculate the total cost.

Table 1. distances of the new data transmission paths for IEEE 118 bus

\begin{tabular}{ccc}
\hline Bus & To Bus & Distance $(\mathrm{k} \mathrm{M})$ \\
\hline 24 & 17 & 16 \\
24 & 31 & 24 \\
24 & 39 & 32 \\
24 & 49 & 28 \\
24 & 96 & 20 \\
5 & 27 & 33 \\
37 & 47 & 16 \\
32 & 43 & 18 \\
45 & 80 & 31 \\
\hline
\end{tabular}

Table 2. distances of the new data transmission paths for IEEE 14 bus

\begin{tabular}{ccc}
\hline Bus & To Bus & Distance $(\mathrm{k} \mathrm{M})$ \\
\hline 5 & 10 & 28.52 \\
6 & 10 & 23.2 \\
4 & 14 & 26.2 \\
7 & 14 & 20.2 \\
\hline
\end{tabular}


Table 3. CCBS per unit cost for IEEE 14 bus

\begin{tabular}{cccc}
\hline Bus No. & CCBS cost & Bus No. & CCBS cost \\
\hline 1 & 2 & 8 & 2.6 \\
2 & 2.2 & 9 & 2.6 \\
3 & 2.3 & 10 & 2 \\
4 & 2 & 11 & 2.7 \\
5 & 2.4 & 12 & 2.1 \\
6 & 2.1 & 13 & 2.3 \\
7 & 2.5 & 14 & 2.4 \\
\hline
\end{tabular}

Table 4. CCBS per unit cost for IEEE 118 bus

\begin{tabular}{cccccccc}
\hline Bus No. & CCBS Cost & Bus No. & CCBS Cost & Bus No. & CCBS Cost & Bus No. & CCBS Cost \\
\hline 1 & 3.719 & 60 & 3.3434 & 31 & 3.1984 & 91 & 2.9697 \\
2 & 2.3641 & 61 & 1533 & 32 & 2.9213 & 92 & 3.3111 \\
3 & 1.9626 & 62 & 2.2164 & 33 & 3.9567 & 93 & 2.1998 \\
4 & 2.2863 & 63 & 2.9469 & 34 & 2.542 & 94 & 2.6452 \\
5 & 3.4149 & 64 & 3.2891 & 35 & 2.542 & 94 & 2.6452 \\
6 & 1.7678 & 65 & 3.8697 & 36 & 3.1778 & 95 & 2.5281 \\
7 & 3.5341 & 66 & 3.0082 & 37 & 3.1812 & 96 & 3.0965 \\
8 & 3.8472 & 67 & 2.6127 & 38 & 2.485 & 97 & 3.422 \\
9 & 1.9138 & 68 & 2.9791 & 39 & 3.3338 & 98 & 3.1374 \\
10 & 2.0987 & 69 & 3.4924 & 40 & 2.1127 & 99 & 1.7917 \\
11 & 2.7317 & 70 & 3.1871 & 41 & 2.2335 & 100 & 3.8055 \\
12 & 2.0737 & 71 & 1.524 & 42 & 2.4873 & 101 & 2.228 \\
13 & 3.681 & 72 & 3.331 & 43 & 2.1265 & 102 & 2.9907 \\
14 & 3.6215 & 73 & 3.2627 & 44 & 2.5577 & 103 & 3.0714 \\
15 & 1.659 & 74 & 1.8533 & 45 & 2.5245 & 104 & 2.1432 \\
16 & 2.1787 & 75 & 3.3286 & 46 & 2.8445 & 105 & 3.3132 \\
17 & 3.7605 & 76 & 2.3377 & 47 & 3.1605 & 106 & 3.9032 \\
18 & 2.5059 & 78 & 3.4523 & 48 & 3.9543 & 107 & 3.3752 \\
19 & 2.2869 & 79 & 1.5871 & 49 & 1.9412 & 108 & 2.8049 \\
20 & 3.1107 & 80 & 3.9971 & 50 & 3.2633 & 109 & 1.9165 \\
\hline
\end{tabular}

Table 5. Flow data

\begin{tabular}{cc}
\hline PMU and CD data flow & $128 \mathrm{kbps}$ \\
\hline PMU and CD frame length & $1 \mathrm{~Kb}$ \\
\hline
\end{tabular}

Table 6. Cost factors values

\begin{tabular}{|c|c|c|c|}
\hline Factor & Rate value & Cost & Per unit cost \\
\hline \multirow[t]{6}{*}{$L_{c r p}$ (IEEE 14 bus) } & $0.3 \mathrm{Mbit} / \mathrm{s}$ & $14 \$ / \mathrm{km}$ & 0.00014 \\
\hline & $0.7 \mathrm{Mbit} / \mathrm{s}$ & $40 \$ / \mathrm{km}$ & 0.0004 \\
\hline & $1.3 \mathrm{Mbit} / \mathrm{s}$ & $60 \$ / \mathrm{km}$ & 0.0006 \\
\hline & 2.7 Mbit/s & $100 \$ / \mathrm{km}$ & 0.001 \\
\hline & $6 \mathrm{Mbit} / \mathrm{s}$ & $200 \$ / \mathrm{km}$ & 0.002 \\
\hline & $200 \mathrm{Mbit} / \mathrm{s}$ & $50000 \$ / \mathrm{km}$ & 0.5 \\
\hline \multirow{9}{*}{$L_{c r p}$ (IEEE 118 bus) } & $0.3 \mathrm{Mbit} / \mathrm{s}$ & $14 \$ / \mathrm{km}$ & 0.00014 \\
\hline & 1.3 Mbit/s & $60 \$ / \mathrm{km}$ & 0.0006 \\
\hline & $6 \mathrm{Mbit} / \mathrm{s}$ & $200 \$ / \mathrm{km}$ & 0.002 \\
\hline & $12 \mathrm{Mbit} / \mathrm{s}$ & $400 \$ / \mathrm{km}$ & 0.004 \\
\hline & $24 \mathrm{Mbit} / \mathrm{s}$ & $800 \$ / \mathrm{km}$ & 0.008 \\
\hline & $48 \mathrm{Mbit} / \mathrm{s}$ & $1600 \$ / \mathrm{km}$ & 0.016 \\
\hline & $96 \mathrm{Mbit} / \mathrm{s}$ & $3200 \$ / \mathrm{km}$ & 0.032 \\
\hline & $600 \mathrm{Mbit} / \mathrm{s}$ & $18000 \$ / \mathrm{km}$ & 0.18 \\
\hline & $1200 \mathrm{Mbit} / \mathrm{s}$ & $36000 \$ / \mathrm{km}$ & 0.36 \\
\hline \multirow[t]{3}{*}{ switch $_{\text {crp }}$ ( IEEE 14 bus) } & $100 \mathrm{Mbit} / \mathrm{s}$ & $10,000 \$ /$ node & 0.1 \\
\hline & $250 \mathrm{Mbit} / \mathrm{s}$ & $20,000 \$ /$ node & 0.2 \\
\hline & $500 \mathrm{Mbit} / \mathrm{s}$ & $40,000 \$ /$ node & 0.4 \\
\hline \multirow{4}{*}{ switch $_{c r p}$ ( IEEE 118 bus) } & $100 \mathrm{Mbit} / \mathrm{s}$ & $10,000 \$ /$ node & 0.1 \\
\hline & $250 \mathrm{Mbit} / \mathrm{s}$ & $20,000 \$ /$ node & 0.2 \\
\hline & $600 \mathrm{Mbit} / \mathrm{s}$ & $40,000 \$ /$ node & 0.4 \\
\hline & $1200 \mathrm{Mbit} / \mathrm{s}$ & $80,000 \$ /$ node & 0.8 \\
\hline \multirow[t]{7}{*}{$P M U_{c h p}$} & 2 channel & $20,000 \$ /$ node & 0.2 \\
\hline & 4 channel & $50,000 \$ /$ node & 0.5 \\
\hline & 6 channel & $60,000 \$ /$ node & 0.6 \\
\hline & 8 channel & $70,000 \$ /$ node & 0.7 \\
\hline & 10 channel & $80,000 \$ /$ node & 0.8 \\
\hline & 12 channel & $90,000 \$ /$ node & 0.9 \\
\hline & 14 channel & $100,000^{\left[1^{*}\right]} \$ /$ node & 1 \\
\hline$P M U_{\text {in }}$ & & $20000 \$ /$ node & 0.2 \\
\hline$\alpha$ (tower cost) & & $1000 \$ / \mathrm{km}$ & $0.01^{[2 *]}$ \\
\hline$L_{\text {in }}$ & & $1000 \$ / \mathrm{km}$ & $0.01^{[2 *]}$ \\
\hline switch $_{\text {in }}$ & & $100 \$ /$ node & $0.001^{[3 *]}$ \\
\hline
\end{tabular}

[1*] This value is used as base for the per unit cost

$\left[2^{*}\right]$ This value is used for all links unless otherwise stated

[3*] This value is used for all nodes unless otherwise stated 


\subsection{Using BPSOGSA Combined with MST}

In this approach, the optimization is divided into three loops as shown in Figure 7. The first loop, the main loop, the BPSOGSA in Section 3 is used to search the best location of the CCBS and PMUs that achieve the observability constraint as shown in Section 2. If the observability condition is not met, the inner loops are not required. Therefore, the following equation is used as the cost function in the outer loop.

$$
\text { Total Cost }=\mathrm{C}_{1}+\text { OBS_Penalty }
$$

where

$\mathrm{OBS}_{\text {Penalty }}=\mathrm{C}_{2} *$ ineqdsum

$C_{1}, C_{2}$ are constants with large value

ineqdsum $=$ summation of all postive elements in $\mathrm{OBS}_{\mathrm{d}}$ vector

$\mathrm{OBS}_{\mathrm{d}}=$ observability right hand side - observability left hand side

Based on $\mathrm{D}_{\text {merged, }}$ the MST or MMST in Section 4 to connect all PMUs, CDs, and CCBS is used in the second loop. The third loop, BPSOGSA is used to allocate the links capacity of the connected network. Cost of the connected network according to (17 This loop return the total) with considering the (20) and (21) as a weighted penalty.

$$
\begin{aligned}
& \max \left(\mathrm{T}_{\mathrm{pmu}}\right)<\mathrm{t}_{\text {req }} \\
& \min \left(\mathrm{R}_{\text {Node }}\right)<\mathrm{r}_{\text {req }}
\end{aligned}
$$

where

$\mathrm{T}_{\mathrm{pmu}}$ is a vector of latency time for all PMUs according to (6)

$\mathrm{R}_{\text {Node }}$ is a vector of latency time for all nodes according to (9), (10).

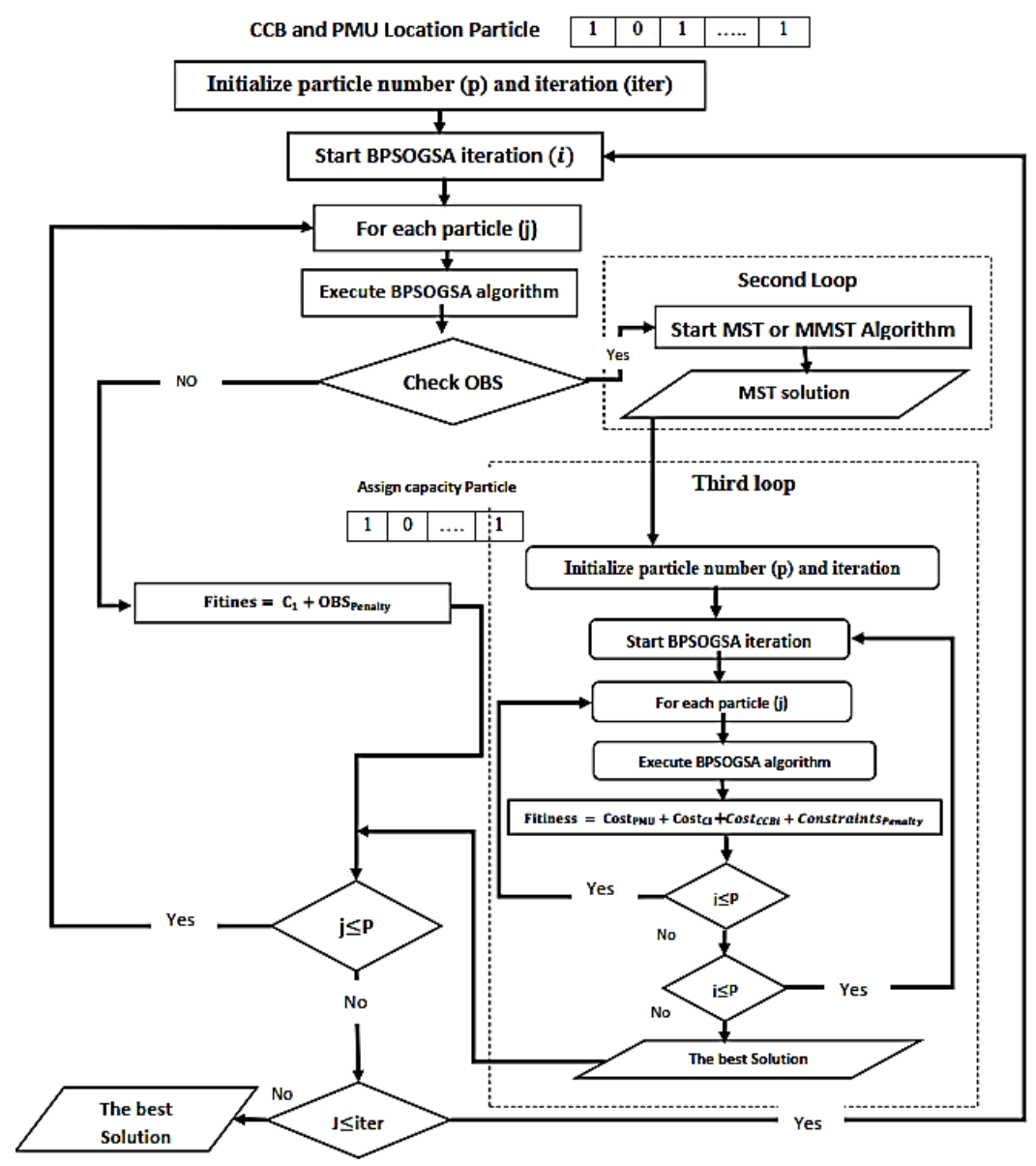

Figure 7. Flow chart of BPSOGSA and MST 


\subsubsection{IEEE 14 bus Case study}

\section{a. Free selection of the CCBS}

Table 7 lists the predefined locations of the PMUs and CDs. In addition, Table 8 shows the results of the proposed approach, and the network topology is shown in Figure 8. The results of the used method in [18] for this case are: cost= 33.048 per unit, maximum latency 0.027844 Sec., and minimum reliability $=0.87146$.

b. Predefined selection of the CCBS $(\mathrm{CCBS}=14)$

Table 9 lists the Predefined locations of the PMUs, CDs, and CCBS. In addition, Table 10 shows the results of the proposed approach, and the network topology is shown in Figure 9. The results of the used method in [18] for this case are: cost= 47.561 per unit, maximum latency 0.02826 Sec., and minimum reliability $=0.83678$.

Table 7. Predefined locations

\begin{tabular}{lc}
\hline PMUs locations & 2,8 \\
\hline CDs locations & 3,10
\end{tabular}

Table 8. Results of the proposed approach

\begin{tabular}{lc}
\hline CCBS location & 10 \\
\hline All PMUs locations & $2,6,8,9$ \\
Total cost (per unit) & 9.212 \\
Maximum latency time (Sec) & 0.028169 \\
Minimum reliability & 0.90821 \\
Runtime (minute) & 40 \\
\hline
\end{tabular}

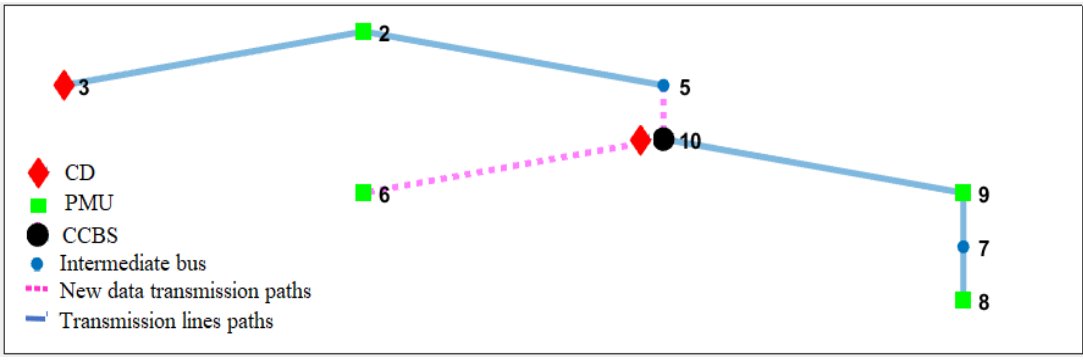

Figure 8 . Network topology (14 bus free selection case)

Table 9. Predefined locations

\begin{tabular}{ll}
\hline PMUs locations & 2,8 \\
CDs locations & 3,10 \\
CCBS location & 14 \\
\hline
\end{tabular}

Table 10. Results of the proposed approach

\begin{tabular}{ll}
\hline All PMUs locations & $2,6,8,9$ \\
Total cost (per unit) & 10.228 \\
Maximum latency time (Sec) & 0.02894 \\
Minimum reliability & 0.88665 \\
Runtime (minute) & 35 \\
\hline
\end{tabular}

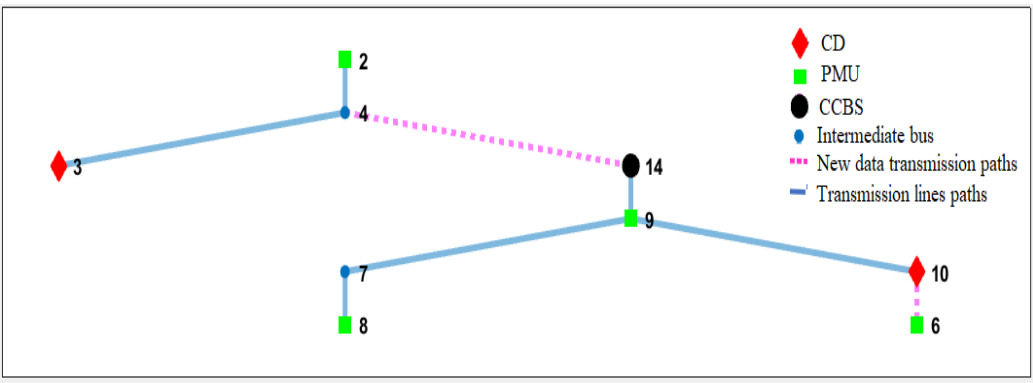

Figure 9. Network topology (14 bus predefined selection case) 


\subsubsection{IEEE 118 bus Case study (Free selection of the CCB)}

The Predefined locations of the PMUs, CDs, and the results of the proposed approach are listed in Table 11. In addition, the network topology is shown in Figure 10. The results of the used method in [18] for this case are: cost $=54$ per unit, maximum latency 0.029074 Sec., and minimum reliability $=0.76225$.

Table 11. Predefined locations and optimization results

\begin{tabular}{|c|c|}
\hline \multicolumn{2}{|r|}{ Predefined locations } \\
\hline PMUs locations & $2,5,10,12,14,21,32,34,37,41,94$ \\
\hline CDs locations & $91,92,96,100,105$ \\
\hline \multicolumn{2}{|r|}{ Results of the proposed approach } \\
\hline CCBS location & 30 \\
\hline All PMUs locations & $\begin{array}{l}2,5,10,11,12,14,17,21,25,29,32,34,37,41,45,49,53,56,62.64,72,73,75,77,80,85,87 \text {, } \\
91,92,94,96,97,100,105,106,110,114,116\end{array}$ \\
\hline Total cost & 49 (Per unit) + penalty of the reliability constraint \\
\hline Maximum latency time (Sec) & 0.031341 \\
\hline Minimum reliability & $0.79868<0.8$ \\
\hline Runtime (minute) & 230 \\
\hline
\end{tabular}

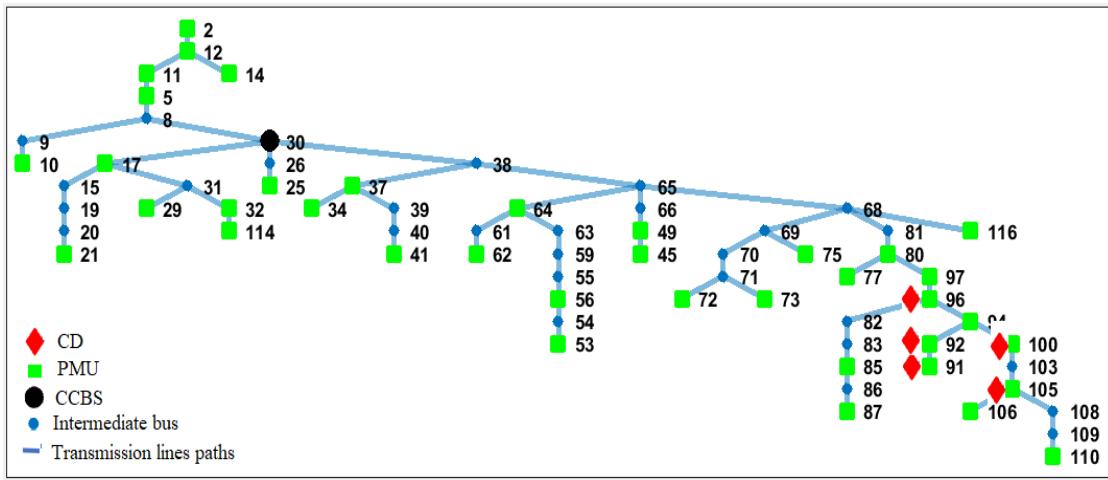

Figure 10. Network topology (118 bus free selection case)

\subsection{Using BPSOGSA}

In this approach, the optimization is divided into three loops.

- The first loop, the main loop, is treated as explained in the Section 7.1

- The second loop, the BPSOGSA with particles dimension equal length of $D_{\text {merged }}$ is used to search the low cost network connection topology, which connect all CCBS, PMUs, and CDs. The value of the fitness function for this loop is evaluated using the connectivity algorithm, which is shown in Figure 11 .

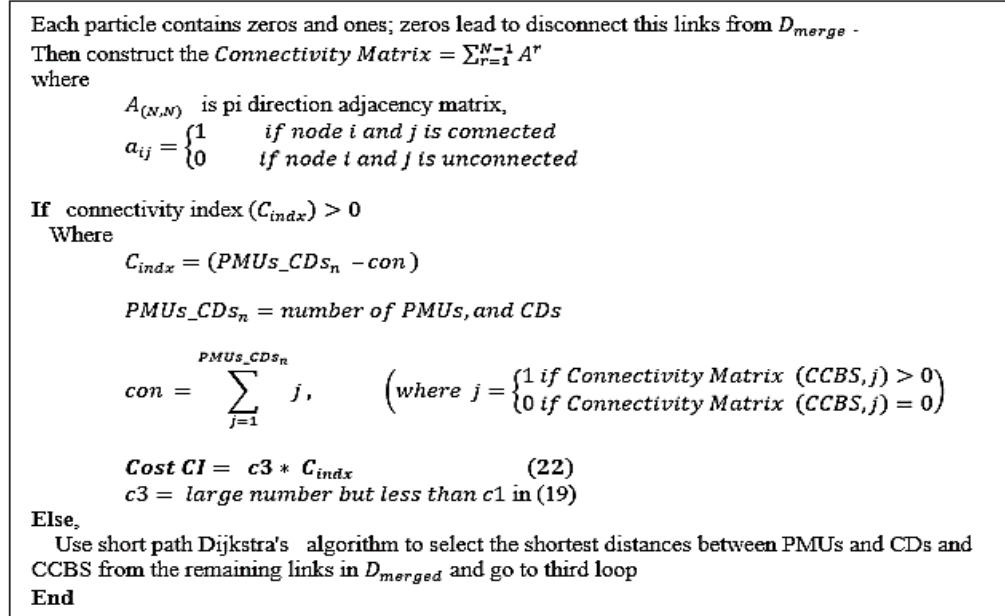

Figure 11. Connectivity algorithm 
- The third loop is treated as explained in the Section 7.1 complete the flowchart of the approach is shown in Figure 12.

The main difference between the approach in this section and the approach in Section 7.1 is that the connection topology is not depend on the length of the network, but the topology connection is depend on the CI cost.

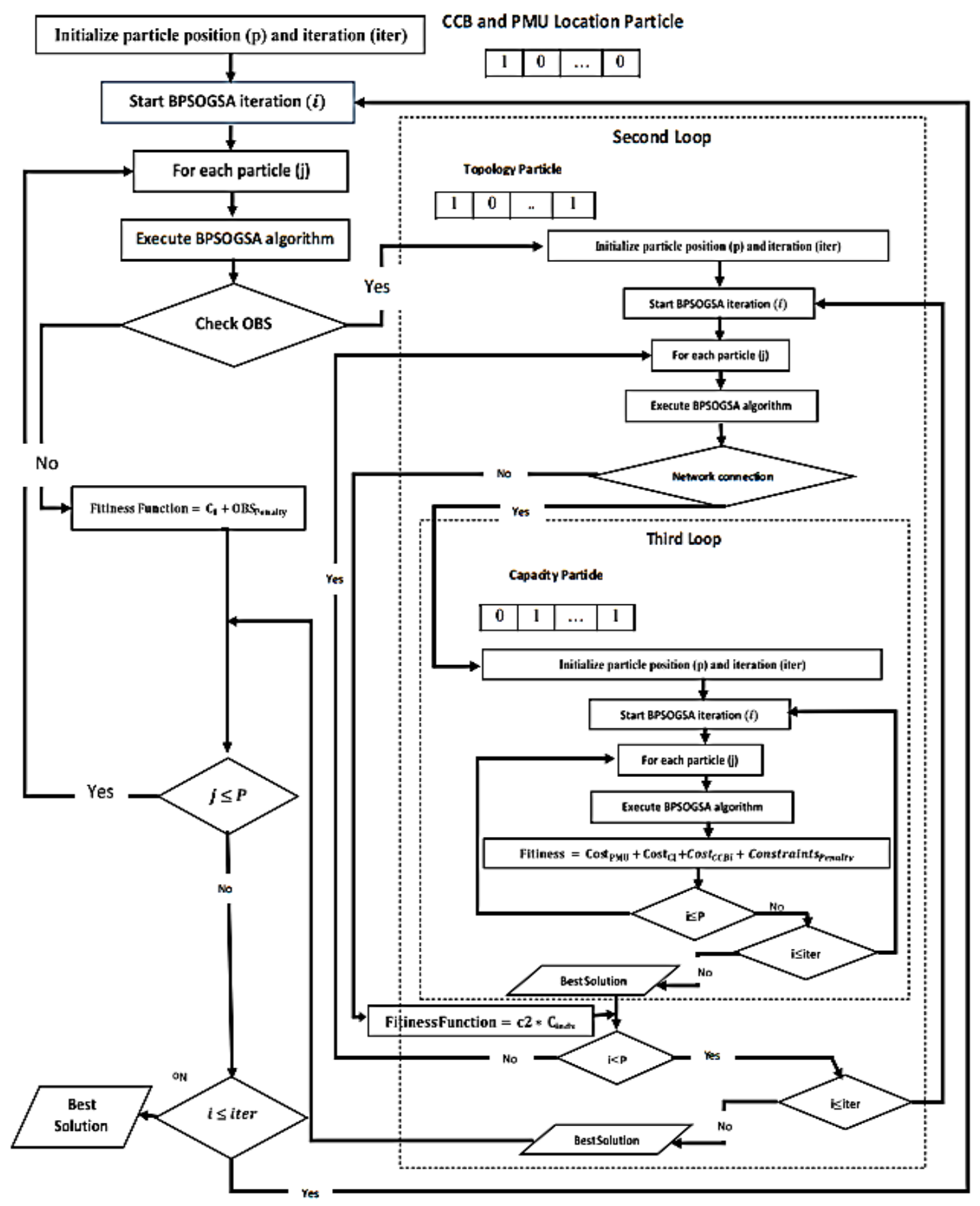

Figure 12. Flow chart of BPSOGSA

\subsubsection{IEEE 14 bus Case study}

a. Free selection of the CCBS

Table 7 lists the Predefined locations of the PMUs and CDs. In addition, Table 12 shows the results of the proposed approach, and the network topology is shown in Figure 13. 
Table 12. Results of the proposed approach

\begin{tabular}{ll}
\hline CCBS location & 10 \\
\hline All PMUs locations & $2,6,8,9$ \\
Total cost (Per unit) & 9.1316 \\
Maximum latency time (Sec) & 0.030168 \\
Minimum reliability & 0.87146 \\
Runtime (minute) & 200 \\
\hline
\end{tabular}

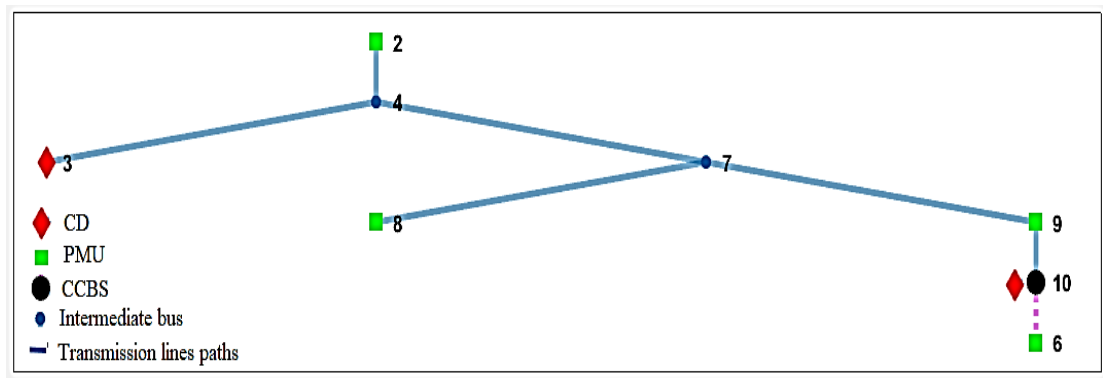

Figure 13. Network topology (14 bus free selection case)

b. Predefined selection of the CCBS $(\mathrm{CCBS}=14)$

Table 9 lists the Predefined locations of the PMUs and CDs. In addition, Table 13 shows the results of the proposed approach, and the network topology is shown in Figure 14.

Table 13. Results of the proposed approach

\begin{tabular}{ll} 
All PMUs locations & $2,6,8,9$ \\
Total cost (Per unit) & 10.074 \\
Maximum latency time (Sec) & 0.028836 \\
Minimum reliability & 0.89372 \\
Runtime (minute) & 180 \\
\hline
\end{tabular}

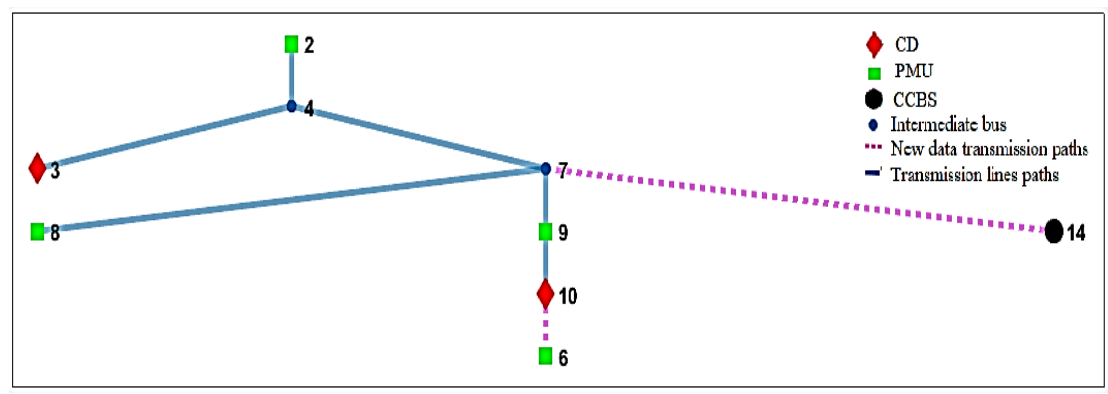

Figure 14. Network topology (118 bus predefined selection case)

\subsubsection{IEEE 118 bus Case study (Free selection of the CCBS)}

Table 11 lists the Predefined locations of the PMUs and CDs. In addition, Table 14 shows the results of the proposed approach, and the network topology is shown in Figure 15.

Table 14. Results of the proposed approach

\begin{tabular}{ll}
\hline CCBS location & 26 \\
All PMUs locations & $2,5,10,12,14,15,17,21,25,29,32,34,37,41,45,49$, \\
& $53,56,62,64,72,73,75,77,80,85,87,91,92,94,100$, \\
& $105,110,114,116$ \\
Total cost (Per unit) & 45.267 \\
Maximum latency time (Sec) & 0.030619 \\
Minimum reliability & 0.80655 \\
Runtime (minute) & 1510 \\
\hline
\end{tabular}

Int J Elec \& Comp Eng, Vol. 10, No. 3, June 2020 : 2824 - 2841 


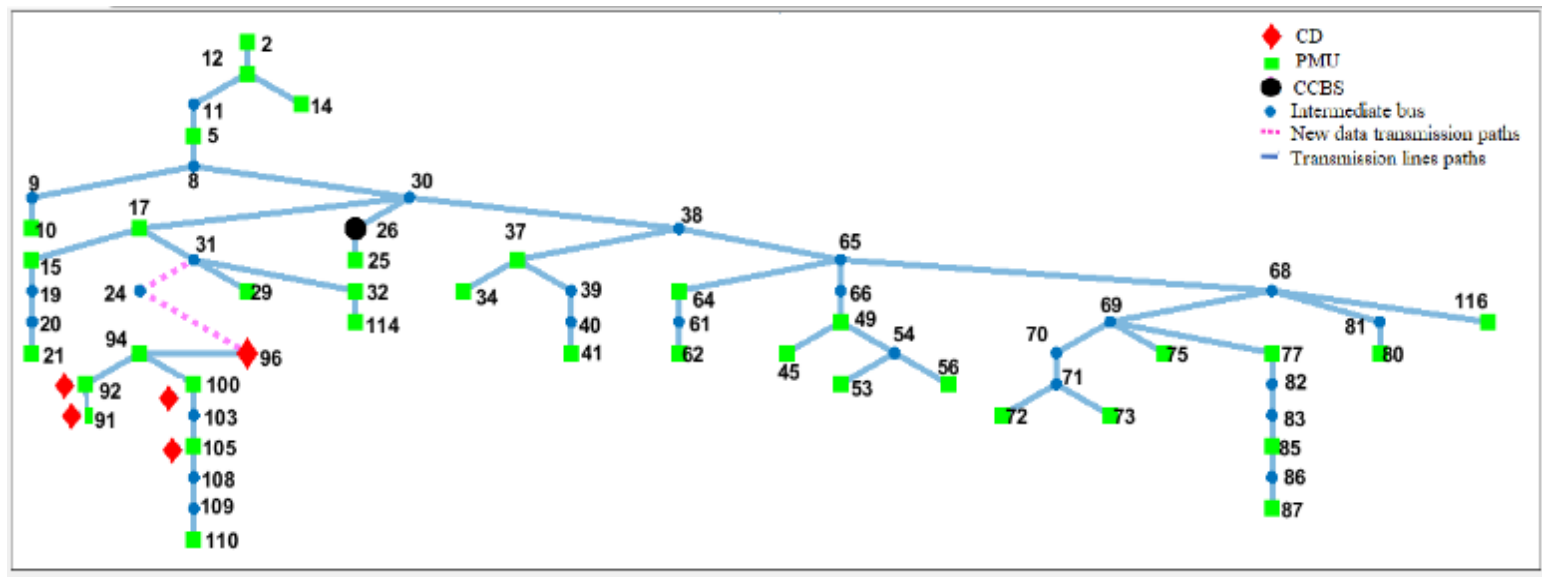

Figure 15. Network topology (118 bus free selection case)

\section{RESULTS DISCUSSION}

The following results were observed from simulation results.

- The run time of second approach is longer than the first approach

- The second approach is more efficient than the first approach, especially if the difference in price resulting from the change in channel capacity is significant.

- Indeed, the methods presented in [18] was unsuccessful to achieve the global solution. Since it used MST algorithm to find the network topology and did not take into account the channel capacity allocation. Also due to, the multi-loop is not used in this method, the runtime is large.

\section{CONCLUSION}

In this study, optimal placement of PMUs and their required CI for power systems are co-optimally designed. Two approaches have been presented. The first approach (i.e. BPSOGSA Combined with MST) and the second approach (i.e. BPSOGSA) to find the optimum placement of PMUs and their CI are investigated using IEEE 14 buses and IEEE 118 buses. The simulation results indicate that the second approach is cost effective. Moreover, the second approach, due to using BPSOGSA in all loops, may succeed converge to the global solution. In contrast, the first approach due to using MST for links topology can take less run time but it may not converge to the global solution. The cost of the CI in this study is not depend on the accumulative length of the OPGW only. However, it considered the switches and the link capacity in the objective function. In addition, the quality of service such as latency time and the reliability of the communication network and the degree of the observability are considered. Also, the partially optimization problem (predefined locations of some PMUs and CDs), and the economic study for additional new data paths are considered in the proposed approaches.

\section{REFERENCES}

[1] J. Ding and Z. Cai, "Mixed Measurements State Estimation Based on Wide-Area Measurement System and Analysis," 2005 IEEE/PES Transmission \& Distribution Conference \& Exposition: Asia and Pacific, Dalian, pp. 1-5, 2005.

[2] P. System, R. Committee, I. Power, and E. Society, "IEEE Guide for Synchronization, Calibration, Testing, and Installation of Phasor Measurement Units (PMUs) for Power System Protection and Control IEEE Power and Energy Society,” IEEE Std C37.242-2013, no. March, pp. 1-107, 6 March 2013.

[3] M. Shahraeini, M. H. Javidi, and M. S. Ghazizadeh, "A new approach for classification of data transmission media in power systems," 2010 International Conference on Power System Technology, Hangzhou, pp. 1-7, 2010.

[4] R. F. Nuqui and A. G. Phadke, "Phasor measurement unit placement techniques for complete and incomplete observability," in IEEE Transactions on Power Delivery, vol. 20, no. 4, pp. 2381-2388, Oct 2005.

[5] J. Gao, Y. Xiao, J. Liu, W. Liang, and C. L. P. Chen, "A survey of communication/networking in Smart Grids," in Future Generation Computer Systems, vol. 28, no. 2, pp. 391-404, 2012.

[6] S. S. S. R. Depuru, L. Wang, and V. Devabhaktuni, "Smart meters for power grid: Challenges, issues, advantages and status," in Renewable and Sustainable Energy Reviews, vol. 15, no. 6, pp. 2736-2742, August 2011.

[7] V. Cagri and F. C. Lambert, "A Survey on Communication Networks for Electric System Automation," in Computer Networks, vol. 50, no. 7, pp. 877-897, 2006.

[8] M. Chenine, K. Zhu, and L. Nordström, "Survey on priorities and communication requirements for PMU-based applications in the nordic region," 2009 IEEE Bucharest PowerTech, Bucharest, pp. 1-8, 2009.

Generalized optimal placement of PMUs considering power system observability ... (M. M. H. Elroby) 
[9] J. De La Ree, V. Centeno, J. S. Thorp, and A. G. Phadke, "Synchronized phasor measurement applications in power systems," in IEEE Transactions on Smart Grid, vol. 1, no. 1, pp. 20-27, Jun. 2010.

[10] B. Gou, "Generalized integer linear programming formulation for optimal PMU placement," in IEEE Transactions on Power Systems, vol. 23, no. 3, pp. 1099-1104, Aug. 2008.

[11] T. L. Baldwin, L. Mili, M. B. Boisen, and R. Adapa, "Power System Observability with Minimal Phasor Measurement Placement," in IEEE Transactions on Power Systems, vol. 8, no. 2, pp. 707-715, May 1993.

[12] A. Enshaee, R. A. Hooshmand, and F. H. Fesharaki, "A new method for optimal placement of phasor measurement units to maintain full network observability under various contingencies," in Electric Power Systems Research, vol. 89, pp. 1-10, Aug. 2012.

[13] B. X. and A. Abur, "Optimal Placement of Phasor Measurement Units for State," PSERC, Final Project Report, pp. 0-3, Oct. 2005.

[14] R. F. Nuqui, "State Estimation and Voltage Security Monitoring Using Synchronized Phasor Measurements," Ph.D. Diss. Dept. Elect. Comput. Eng., Virginia Polytech. Inst. State Univ., Blacksburg, VA, pp. 1-206, 2001.

[15] E. Abiri, F. Rashidi, and T. Niknam, "An optimal PMU placement method for power system observability under various contingencies," International Transactions on Electrical Energy Systems, vol. 25, no. 4, pp. 589-606, 2015.

[16] B. Xu, A. Abur, "Observability analysis and measurement placement for systems with PMUs," IEEE PES Power Systems Conference and Exposition, New York, NY, vol. 2, pp. 943-946, 2004.

[17] G. N. Korres, P. J. Katsikas, K. a. Clements, and P. W. Davis, "Numerical observability analysis based on network graph theory," in IEEE Transactions on Power Systems, vol. 18, no. 3, pp. 1035-1045, Aug. 2003.

[18] M. Shahraeini, M. S. Ghazizadeh, and M. H. Javidi, "Co-optimal placement of measurement devices and their related communication infrastructure in wide area measurement systems," in IEEE Transactions on Smart Grid, vol. 3, no. 2, pp. 684-691, June 2012.

[19] I. E. C. 870-6-1-1995, "Technical Report on Telecontrol Equipment and 29 Systems - Part 6," ISBN 978-2-83220919-6 1995.

[20] X. Tai, D. Marelli, E. Rohr, and M. Fu, "Optimal PMU placement for power system state estimation with random component outages," International Journal of Electrical Power \& Energy Systems, vol. 51, pp. 35-42, Oct. 2013.

[21] V. Khiabani and K. Farahmand, "Max Covering Phasor Measurement Units Placement for Partial Power System Observability," Engineering Management Research, vol. 2, no. 1, pp. 43-54, 2013.

[22] O. Gomez, G. Anders, and M. A. Rios, "Reliability-based phasor measurement unit placement in power systems considering transmission line outages and channel limits," in IET Generation, Transmission \& Distribution, vol. 8, no. 1, pp. 121-130, Jan. 2014.

[23] F. Aminifar, A. Khodaei, M. Fotuhi-Firuzabad, and M. Shahidehpour, "Contingency-constrained PMU placement in power networks," in IEEE Transactions on Power Systems, vol. 25, no. 1, pp. 516-523, Feb. 2010.

[24] V. Khiabani, E. Erdem, K. Farahmand, and K. Nygard, "Genetic algorithm for instrument placement in smart grid," 2013 World Congress on Nature and Biologically Inspired Computing, Fargo, ND, pp. 214-219, 2013.

[25] S. F. Marin FJ, Garcia-Lagos F, Joya G, "Genetic algorithms for optimal of SID placement of phasor measurement units in electrical networks," in Electronics Letters, vol. 39, no. 19, pp. 1403-1405, 2003.

[26] L. Mili, T. Baldwin, and R. Adapa, "Phasor measurement placement for voltage stability analysis of power systems 29th IEEE Conference on Decision and Control, Honolulu, HI, USA, pp. 3033-3038 vol. 6, 1990.

[27] J. Peng, Y. Sun, and H. F. Wang, "Optimal PMU placement for full network observability using Tabu search algorithm," International Journal of Electrical Power \& Energy Systems, vol. 28, no. 4, pp. 223-231, May 2006.

[28] M. M. Ahmadi A, Alinejad-Beromi Y, "Optimal PMU placement for power system observability using binary particle swarm optimization and considering measurement redundancy," Expert Systems with Applications, vol. 38, no. 6, pp. 7263-7269, Jun. 2011.

[29] B. Gou, "Optimal placement of PMUs by integer linear programming," in IEEE Transactions on Power Systems, vol. 23, no. 3, pp. 1525-1526, Aug. 2008.

[30] S. Mirjalili and S. Z. M. Hashim, "A new hybrid PSOGSA algorithm for function optimization," 2010 International Conference on Computer and Information Application, Tianjin, pp. 374-377, 2010.

[31] S. Mirjalili, G.-G. Wang, and L. dos S. Coelho, "Binary optimization using hybrid particle swarm optimization and gravitational search algorithm," in Neural Computing and Applications, vol. 25, no. 6, pp. 1423-1435, 2014.

[32] M. Newman, Networks: an introduction. Oxford university press, ISBN-13: 9780199206650, Mar. 2010.

[33] E. W. Dijkstra, “A Note on Two Problems in Connection with Graphs," J. Numerische Mathematik, vol. 1, no. 1 pp. 269-271, Dec. 1959.

[34] M. Sniedovich, "Dijkstra's algorithm revisited: the dynamic programming connection," J. Control and cybernetics, vol. 35, no. 3, pp. 599-620, Jan. 2006.

[35] M. Ingram, D. Hart, D. Novocel, M Subramanian, "Real-time wide area measurements for adaptive protection and control," Proc. Nat. Sci., 2000.

[36] L. Lin and M. Gen, "Node-based genetic algorithm for communication spanning tree problem," in IEICE Transactions on Communications, vol. E89-B, no. 4, pp. 1091-1098, Apr. 2006.

[37] J.-B. Jo,. J. R. Kim, J. U. Lee, "Hierarchical Spanning Tree Network Design with Nash Genetic Algorithm," Computers \& Industrial Engineering, vol. 56, no. 3, pp. 1040-1052, Apr. 2009.

[38] "Real time Application of Synchophasors for improving Reliability," NERC Rep., vol. Oct. 2010, pp. 77, 2010.

[39] M. Chenine, I. Al Khatib, J. Ivanovski, V. Maden, and L. Nordstrom, "PMU traffic shaping in IP-based wide area communication," 2010 5th International Conference on Critical Infrastructure (CRIS), Beijing, pp. 1-6. 2010. 
[40] H. Pucha, Y. Zhang, Z. M. Mao, and Y. C. Hu, "Understanding network delay changes caused by routing events," in ACM SIGMETRICS Performance Evaluation Review, vol. 35, no. 1, pp. 73, Jun. 2007.

[41] M. Gerla, J. A. Suruagy Monteiro and R. Pazos, "Topology design and bandwidth allocation in ATM nets," in IEEE Journal on Selected Areas in Communications, vol. 7, no. 8, pp. 1253-1262, Oct. 1989.

[42] G. Hill, "The Cable and Telecommunications Professionals," Focal Press, vol. 2, ISBN 9780240807485 - CAT\# Y140750, pp. 462, August 2008.

[43] P. System, R. Committee, I. Power, and E. Society, "IEEE Standard for Synchrophasor Data Transfer for Power Systems," in IEEE Std C37.118.2-2011 (Revision of IEEE Std C37.118-2005), vol., no., pp.1-53, 28 Dec. 2011.

[44] T. S. Sang Ahn, Meejeong Lee, T. S. S Ahn, M Lee, and L. and S. Ahn, "Topology reconfiguration of an IP network embedded over an ATM network," IEICE TRANS. COMMUN, vol. E84-B, no. 11, Nov. 2001.

[45] R. Billinton and R. A. Allan, "Reliability Evaluation of Engineering Systems: Concepts and Techniques," pp. 469, ISBN 978-1-4899-0685-4, 1992.

[46] M. S. Ghazizadeh, M. Shahraeini, M. H. Javidi, "Comparison between communication infrastructures of centralized and decentralized wide area measurement systems," in IEEE Transactions on Smart Grid, vol. 2, no. 1, pp. 206-211, Mar. 2011.

[47] S. Akhlaghi, N. Zhou, and N. E. Wu, "PMU placement for state estimation considering measurement redundancy and controlled islanding," 2016 IEEE Power and Energy Society General Meeting (PESGM), Boston, MA, pp. 1-5, 2016.

[48] F. Aminifar, M. Fotuhi-Firuzabad, A. Safdarian, A. Davoudi, and M. Shahidehpour, "Synchrophasor Measurement Technology in Power Systems: Panorama and State-of-the-Art,” in IEEE Access, vol. 2, pp. 1607-1628, 2014. 